\section{Resultados de la vacunación antimeningocócica en Cuba}

La septicemia y la meningitis son enfermedades meningocócicas invasoras (EMI) que plantean un gran peligro para la vida humana. En total se han identificado 13 serogrupos causales de enfermedad, de los cuales los A, B y C son los más comunes y producen $90 \%$ de todos los casos de meningitis meningocócica en el mundo. En Cuba, las EMI eran endémicas y no se consideraban un problema de salud. Entre 1962 y 1978, la incidencia de casos aumentó de 0,1 a 1,8 por 100000 habitantes, debido principalmente a un incremento de la incidencia de infección por meningococos del grupo C. En vista de la situación, en 1979 el Ministerio de Salud Pública optó por realizar una campaña nacional de vacunación con una vacuna a base de polisacáridos capsulares de los serogrupos A-C (Laboratorios Mérieux). La campaña, que se dirigió a todos los niños de 3 meses a 19 años de edad, logró una cobertura de $80 \%$ y condujo a una reducción de las infecciones por meningococos del seogrupo C. No obstante, la incidencia de meningitis meningocócica siguió aumentando y en 1980 el Ministerio declaró que las EMI constituían un problema de salud de primera magnitud en el país. Predominaban los serogrupos B $(78,4 \%)$ y C $(7,2 \%)$. La incidencia alcanzó su pico máximo en 1983, con 14,4 casos por 100000 habitantes.

En los años ochenta se emprendió en Cuba el estudio de una vacuna contra meningococos de los serogrupos B y C (VA-MENGOC-BC) elaborada a partir de la cepa Cu385/83 (B:4; P1.15). Esta vacuna contenía polisacáridos del grupo $\mathrm{C}$ y proteínas de la membrana externa a las que se les habían extraído los lipooligosacáridos, y estaba enriquecida con proteínas capsulares de 65 a $95 \mathrm{kDa}$. Tras la obtención de resultados alentadores en las pruebas de eficacia realizadas en 1987 y 1988, el Ministerio de Salud Pública llevó a cabo en 1989 una campaña de vacunación dirigida a toda la población menor de 20 años y logró una cobertura mayor de 95\%. A partir de 1991, la nueva vacuna se incluyó en el Programa Nacional de Inmunización (PNI) para todos los lactantes (una primera dosis a los 3,5 meses de edad y la segunda a los 5,5 meses). Desde entonces se ha logrado una cobertura sostenida de $89,4 \%$.

Recientemente se efectuó un estudio para comparar la incidencia de EMI antes y después de la campaña nacional de vacunación de 1989. En un mapa del territorio nacional se marcó para cada municipio la densidad de la incidencia (DI) correspondiente a distintos períodos antes y después de la campaña y se detectaron diferencias muy notables (antes: DI $>8,8 / 10^{5}$ años-persona; después: $\mathrm{DI}<6,5 / 10^{5}$ años-persona). Las diferencias porcentuales detectadas al comparar la DI al comienzo y al final de cada período fueron mucho mayores después de la campaña de vacunación $(87 \%)$ que antes de ella (37\%). Estas diferencias fueron significativas en los grupos de edad vacunados. Se observó un descenso de la DI en casi todas las municipalidades y algunas no notificaron ningún caso de EMI. La técnica de "mapeo" empleada ilustra el impacto homogéneo que tuvo en todo el país la campaña de inmunización con la vacuna VAMENGOC-BC. Después de la campaña, la morbilidad por meningococos del serogrupo B comenzó a declinar en niños de 1 año y menores. La frecuencia de casos de EMI en el territorio nacional fue significativamete mayor en los grupos poblacionales que no habían sido vacunados y no se produjo ningún brote de enfermedad tras la campaña de vacunación. Estos datos confirman el efecto protector de la vacuna aplicada a la población menor de 20 años.

Aunque en otros países con epidemias de EMI la VA-MENGOC-BC se ha mostrado poco eficaz contra meningococos del serogrupo $B$, particularmente en niños menores de 5 años, en Cuba se observó un marcado descenso de la epidemia después de la aplicación en masa de la vacuna contra serotipos específicos, seguida de la inmunización sistemática. Estos resultados coinciden con los de estudios efectuados en Cuba en años anteriores, en los cuales se observó una eficacia de 93 a $98 \%$ en niños de 0 a 5 años. Cabe resaltar que la estrategia preventiva del Ministerio de Salud Pública de aplicar la VA-MENGOC-BC a todos los niños menores de 1 año como parte del PNI, práctica que se inició en 1991, ha sido un factor de gran importancia en el mantenimiento de la reducción de la morbilidad, aun habiéndose confirmado la presencia de meningococos causales circulantes. (Pérez Rodríguez A, Dickinson F, Baly A, Martinez $\mathrm{R}$. The epidemiological impact of antimeningococcal B vaccination in Cuba. Mem Inst Oswaldo Cruz 1999;94(4):433-440.) 


\section{La inmunización de recién nacidos prematuros o de bajo peso}

En lactantes, el sistema inmunitario está menos desarrollado que en adultos y niños mayores. La inmunoglobulina $G$ de la madre se transfiere al feto por la vía transplacentaria y las concentraciones fetales de IgG guardan relación con su edad gestacional. Como resultado, los niños prematuros son más susceptibles a las infecciones propias de la niñez que los niños que nacen a término y tienen, además, un mayor riesgo de morir a causa de sus complicaciones. A fin de controlar la morbilidad y mortalidad asociadas con las enfermedades infantiles que pueden prevenirse por vacunación, el Comité Asesor en Enfermedades Infecciosas de los Centros para el Control y la Prevención de Enfermedades (CDC) en Atlanta, Georgia, recomienda que los niños que nacen prematuramente o con bajo peso sean vacunados a las mismas edades que los niños que nacen a término.

Algunos estudios han revelado, sin embargo, que los trabajadores de la salud no siempre observan esta recomendación y que vacunan más tarde a los niños prematuros y de bajo peso debido a la creencia errónea de que estos niños aún no pueden montar una respuesta inmunitaria satisfactoria. De ahí que un grupo de investigadores se propusiera investigar las prácticas de inmunización actuales e identificar los factores que se asocian con el riesgo de que los niños no sean vacunados a su debido tiempo. El estudio, que se basó en un extenso conjunto de datos tomados de la población estadounidense, se efectuó en un total de 11580 niños que nacieron prematuros o con bajo peso. Todos los niños fueron seguidos desde el nacimiento hasta los 2 meses de edad y 6832 de ellos fueron seguidos adicionalmente hasta los 24 meses. A la edad de 2 meses había 173373 niños de peso normal y nacidos a término en el grupo de testigos y a los 24 meses, 103324.

Se determinó si cada niño estaba al día en el esquema de vacunación, tomándose como fecha límite el último día del período de edad del niño en meses. Por ejemplo, un niño se consideraba vacunado a los 2 meses de edad, como corresponde, si había recibido la vacuna antes de los 91 días de nacido. Las variables de resultado fueron el estado de inmunización específico de la edad en función del tiempo de gestación y del peso al nacer $(<1500 \mathrm{~g}$; 1500 a $2500 \mathrm{~g} ;<38$ semanas de gestación con peso al nacer $>2500 \mathrm{~g}, \mathrm{y}$ nacido a término con peso normal) y las características de los pacientes que mostraban una asociación con estar al día en el esquema de inmunización.

En todas las edades, los niños que nacieron con un peso menor de $1500 \mathrm{~g}$ estuvieron al día en sus esquemas de vacunación con menos frecuencia que los otros niños. A los 6 meses, de 52 a $65 \%$ de los niños nacidos con un peso menor de $1500 \mathrm{~g}$ estaban al día en sus inmunizaciones, en comparación con 69 a 73\% de los niños que habían nacido con un peso de 1500 a 2500 g; 66 a $80 \%$ de los niños prematuros que pesaban más de 2500 g, y 65 a $76 \%$ de los niños nacidos a término y con un peso normal. A los 24 meses de edad, 78 a $86 \%$ de los niños que habían nacido con un peso menor de 1500 g ya estaban al día en su esquema de inmunización, frente a 84 a $89 \%$ de los niños nacidos con un peso mayor.

Estos resultados indican que, en los Estados Unidos de América, los niños que nacen prematuramente reciben sus vacunas más o menos a las mismas edades que la población en general, mientras que los niños que nacen con un peso muy bajo manifiestan un pequeño atraso en sus esquemas de vacunación pero tienden a ponerse al día antes de cumplir los 24 meses de edad. (Davis RL, Rubanowice D, Shinefield HR, Lewis N, Gu D, Black SB, et al. Immunization levels among premature and lowbirth-weight infants and risk factors for delayed upto-date immunization status. JAMA 1999;282(6): 547-553.)

\section{La tolerancia a la glucosa y su asociación con la mortalidad}

Según la Asociación Estadounidense de Diabetes (American Diabetes Association, ADA), los resultados de la prueba de glucosa en ayunas son suficientes para diagnosticar la diabetes mellitus. En cambio, la Organización Mundial de la Salud (OMS) recomienda basar el diagnóstico en la glucemia obtenida 2 horas después de una carga oral de $75 \mathrm{~g}$ de glucosa. Un grupo de investigadores se propuso examinar la mortalidad asociada con los criterios diagnósticos de ambas entidades, para lo cual reunió los resultados de 13 estudios de cohorte prospectivos realizados en Europa. En total los datos provinieron de 18048 hombres y 7316 mujeres de 30 años de edad o mayores. El seguimiento promedio de cada persona fue de 7,3 años.

En comparación con los hombres cuya glucemia en ayunas era normal $(<6,1 \mathrm{mmol} / \mathrm{L})$, los hombres con diabetes mellitus recién diagnosticada según los criterios de la ADA (glucosa en ayunas $\geq 7,0$ $\mathrm{mmol} / \mathrm{L})$ tuvieron un riesgo relativo de morir de 1,81 (IC95\%: 1,49 a 2,20); para las mujeres, este riesgo relativo fue de 1,79 (IC95\%: 1,18 a 2,69). Para los casos con alteración de la glucemia en ayunas (6,1-6,9 mmol/L), los riesgos relativos fueron de 1,21 (IC95\%: 1,05 a 1,41) y de 1,08 (IC95\%: 0,70 a 1,66$)$. En el caso de los criterios de la OMS $(\geq 11,1$ $\mathrm{mmol} / \mathrm{L})$, los riesgos relativos para casos recién 
diagnosticados de diabetes mellitus fueron de 2,02 (IC95\%: 1,66 a 2,46) en hombres y de 2,77 (IC95\%: 1,96 a 3,92) en mujeres $y$, para los casos con alteración de la tolerancia a la glucosa $(7,8$ a 11,1 mmol/L), estos riesgos relativos fueron de 1,51 (IC95\%: 1,32 a 1,72 ) y 1,60 (IC95\%: 1,22 a 2,10). Para cada categoría de glucemia en ayunas se observó un aumento de la mortalidad en asociación con un incremento de la glucemia 2 horas después de la carga oral. No obstante, la glucemia en ayunas no mostró ninguna tendencia ascendente en personas con resultados anormales en la prueba de tolerancia o con un diagnóstico de diabetes arrojado por esta prueba.

A juzgar por los resultados obtenidos en este estudio, las concentraciones de glucosa en ayunas no permiten identificar a personas hiperglucémicas que están en mayor riesgo de morir. La prueba oral de tolerancia a la glucosa proporciona información adicional que tiene un valor pronóstico y que permite detectar a aquellos individuos que tienen alterada la tolerancia a la glucosa, en quienes se observa un mayor riesgo atribuible de morir. (Grupo de estudio DECODE, por el European Diabetes Epidemiology Group. Lancet 1999;354:617-621.)

\section{Índice de masa corporal y cáncer de colon en adultos estadounidenses}

Pese al gran número de estudios prospectivos y de casos y testigos que se han llevado a cabo sobre el tema, aún faltan datos que permitan determinar si la obesidad constituye o no un factor de riesgo de cáncer de colon, especialmente en la mujer. Algunos consideran que la obesidad es indicadora indirecta de un consumo excesivo de alimentos, una vida sedentaria u otro factor no cuantificable. Dado su marcado aumento desde los años setenta, es importante determinar si la obesidad conlleva riesgos de morbilidad o mortalidad, entre ellos el de padecer cáncer de colon.

Un investigador se propuso estudiar la asociación entre obesidad y cáncer de colon en una cohorte de hombres y mujeres de 25 a 74 años, representativa de la población estadounidense en general, que participó en la Primera Encuesta de Salud y Nutrición (National Health and Nutrition Survey) de 1971 a 1975 y que tuvo un seguimiento hasta 1992. Entre las 13420 personas incluidas en la muestra analizada, se encontraron 222 casos de cáncer de colon. La estatura y el peso de toda la muestra se midieron durante el examen físico preliminar.

En comparación con individuos cuyo índice de masa corporal (IMC) era menor de $22 \mathrm{~kg} / \mathrm{m}^{2}$, las personas con un IMC de $22 \mathrm{a}<24 \mathrm{~kg} / \mathrm{m}^{2}, 24$ a $<26$ $\mathrm{kg} / \mathrm{m}^{2}, 26$ a $<28 \mathrm{~kg} / \mathrm{m}^{2}, 28$ a $<30 \mathrm{~kg} / \mathrm{m}^{2}$, y $\geq 30$ $\mathrm{kg} / \mathrm{m}^{2}$ mostraron los siguientes riesgos relativos, respectivamente: 1,79 (IC95\%: 0,87 a 3,71); 1,86 (IC95\%: 0,86 a 4,03); 2,47 (IC95\%: 1,14 a 5,32); 3,72 (IC95\%: 1,68 a 8,22), y 2,79 (IC95\%: 1,22 a 6,35). Los riesgos relativos fueron similares en hombres y mujeres. Se encontró, además, una asociación directa entre el grosor del pliegue cutáneo en la zona subescapular y la incidencia de cáncer de colon en hombres pero no en mujeres, aun después de ajustar el efecto del IMC y de otros posibles factores de confusión. En cambio, no se detectó ninguna asociación entre el grosor del pliegue cutáneo del tríceps y el cáncer de colon. También se encontró en este estudio que la fracción poblacional de cáncer de colon atribuible a la obesidad era de $53 \%$ en personas de 25 a 74 años con un IMC $\geq 22 \mathrm{~kg} / \mathrm{m}^{2}$. La población de referencia fue la de personas con un $\mathrm{IMC} \leq 22 \mathrm{~kg} / \mathrm{m}^{2}$.

Los resultados de esta investigación respaldan la hipótesis de que un peso corporal excesivo es factor de riesgo de cáncer de colon, tanto en hombres como en mujeres.

(Ford ES. Body mass index and colon cancer in a national sample of adult US men and women. Am J Epidemiol 1999;150(4):390-398).

\section{El paciente con tendencias suicidas: características generales}

Es frecuente en un hospital general que el psiquiatra evalúe y trate a pacientes jóvenes que han intentado el suicidio, que constituye actualmente una de las principales causas de muerte entre la juventud de diversos países. En 30 a 50\% de los casos de suicidio el paciente ha hecho un intento anterior. De ahí la importancia de caracterizar con precisión los factores asociados con esta tendencia, a fin de identificar los grupos vulnerables y diseñar intervenciones preventivas.

En un hospital general privado de México, D.F., se realizó un estudio prospectivo de dos años de duración (1994 a 1996) de los pacientes que acudieron al servicio de urgencias o directamente al de psiquiatría por intento de suicidio, que se definió como cualquier conducta de autoagresión claramente destinada a conseguir la muerte. Durante ese período se entrevistaron 147 pacientes (112 mujeres, 35 hombres) mediante un cuestionario aplicado en el momento de la consulta al paciente o a sus familiares para recolectar la siguiente información: datos demográficos; antecedentes psiquiátricos según los criterios de clasificación del DSM-III-R; antecedentes médicos de importancia; descripción y mecanismo del intento, intentos previos y causa (manifestada espontáneamente por el propio paciente); examen físico y mental en el momento de la entre- 
vista; servicio del hospital al que fue destinado el paciente por los psiquiatras de la sala de urgencias.

Se encontró una edad promedio de 32,7 años entre las mujeres y de 48,5 años entre los hombres, con una diferencia significativa entre ambos sexos $(P<0,001)$. En lo que respecta al estado civil, $40 \%$ de los pacientes estaban casados, $36,7 \%$ eran solteros, $\mathrm{y}$ el resto eran viudos o divorciados. No obstante, estos dos últimos grupos consuman el suicidio con mayor frecuencia que los otros dos. Por tratarse de una institución privada, la mayoría de los pacientes tenían un nivel educativo alto: $36,6 \%$ de las mujeres y $25,7 \%$ de los hombres habían cursado estudios profesionales. No obstante, se observó una frecuencia de desempleo de $20 \%$ en mujeres y $43 \%$ en hombres. Cuatro pacientes tenían dolor crónico (en dos casos por cáncer), aunque ninguno relacionó su padecimiento con el intento de suicidio. Las mujeres mostraron como antecedentes médicos más frecuentes un foco irritativo en el EEG $(27,2 \%)$ e hipotiroidismo (16\%) y los hombres, diabetes mellitus $(15,6 \%)$ y prostatitis $(15,6 \%)$. Entre los antecedentes psiquiátricos, la depresión fue el más frecuente (61\%), seguido por los trastornos de la personalidad $(16 \%)$, la dependencia del alcohol $(7,4 \%)$ y la esquizofrenia $(4,8 \%)$. Hubo un promedio de 3,3 intentos de suicidio previos, con una diferencia significativa entre hombres y mujeres. El intento de suicidio fue manifestado abiertamente por $63 \%$ de los pacientes; en el resto de los casos el intento fue corroborado por la familia. Solamente cinco pacientes dejaron carta suicida.

El mecanismo de intento más frecuente fue el consumo de una sobredosis de medicamentos $(87 \%)$, principalmente las benzodiazepinas, seguido de arma blanca $(37 \%)$, arma de fuego $(25,7 \%)$, lanzamiento al vacío $(3,6 \%)$, y otros. En general, los métodos más violentos fueron más frecuentes entre los hombres. En 84 pacientes el motivo manifestado fue un conflicto de pareja o familiar. Otras causas menos frecuentes fueron el fracaso escolar, problemas económicos, el duelo, la soledad y el vacío existencial.

Estos resultados coinciden en general con los de otros estudios. No obstante, llama la atención la frecuencia en las mujeres de trastornos tiroideos, que podrían asociarse con alteraciones afectivas y en algunos casos con el consumo de litio. Por otra parte, y a diferencia de lo hallado en otros estudios, la frecuencia general de depresión fue similar en los hombres y las mujeres - normalmente se observa una mayor frecuencia en estas-y no se observó un predominio de personas viudas o solteras entre los casos de intento de suicidio.

Es común la repetición de la conducta suicida y 12 a 15\% de quienes reinciden son enviados al mismo hospital en el curso de un año, muchos con trastornos psiquiátricos documentados. La comunidad médica aún no puede predecir en la población en general qué personas están en riesgo de tratar de suicidarse; sin embargo, el poder definir, mediante una valoración lenta y cuidadosa, las características generales de los pacientes suicidas que acuden a cada institución permite crear estrategias para la prevención de recidivas. (Castelli Candia P, Serrano Gómez C. Caracterización del paciente que acude por intento suicida a un hospital general. Acta Psiquiat Psicol Am Lat 1998;44(3)268-277).

\section{Posible estrategia terapéutica contra las enfermedades degenerativas del cerebro}

Estudios de laboratorio sobre enfermedades cerebrales causadas por priones, tales como el kuru y la enfermedad de Creutzfeldt-Jakob en el ser humano y la llamada "enfermedad de las vacas locas", han arrojado indicios muy claros y sorprendentes sobre lo que podría constituir una estrategia terapéutica para prevenir estas enfermedades. En el número de agosto de la Journal of Virology, científicos del Instituto Nacional de Alergias y Enfermedades Infecciosas (NIAID) de los Estados Unidos de América y sus colegas en Francia y en el Reino Unido notificaron que la presencia de un pequeño fragmento de proteína de origen priónico $(\operatorname{PrP})$ impide que la proteína más grande se pliegue inadecuadamente. Ya que una aberración en el plegamiento de esta proteína se asocia con las enfermedades priónicas, impedir que la proteína del prion adopte una configuración anormal puede ser un elemento clave para frenar la progresión hacia la enfermedad manifiesta.

Las enfermedades provocadas por priones, denominadas encefalopatías espongiformes transmisibles (EET), son trastornos mortales que confieren al cerebro el aspecto de queso suizo debido a la formación de grandes vacuolas detectables en la autopsia. A mediados de los años noventa, estas enfermedades llamaron la atención a nivel mundial cuando en Gran Bretaña se detectó una epidemia de un trastorno degenerativo en 34 seres humanos por consumo de carne procedente de "vacas locas", es decir, vacas con encefalopatía espongiforme bovina. Aunque se produce la transmisión de los bovinos a los seres humanos, la infección suele limitarse a la especie animal donde se origina.

Investigadores en los laboratorios del NIAID en el estado de Montana han demostrado, por ejemplo, que una PrP anormal obtenida de un ratón no puede hacer que una PrP normal obtenida de un hámster adquiera una configuración anormal. No obstante, la PrP de dos tipos de ratones y de háms- 
ter comparten una misma región nuclear capaz de inhibir la conversión de PrP normal a PrP anormal en ambas especies de ratones. Se trata, por consiguiente, de un péptido con la capacidad para inhibir la conversión de las proteínas priónicas en una gran variedad de animales. Aún no se conoce bien el mecanismo inhibidor, pero la importancia de la reacción radica en su posible aplicación para frenar el proceso patológico. Usando células infectadas obtenidas de ratones, los investigadores bloquearon la producción de PrP nuevas anormales al incubar las células afectadas con el péptido nuclear.

Las alteraciones morfológicas de las proteínas que se observan en las enfermedades priónicas impiden que la proteína anormal sea metabolizada por el organismo. Las proteínas resistentes se acumulan y forman placas insolubles que producen problemas neurológicos. Estos efectos pueden demorar muchos años e incluso decenios en aparecer y han obstaculizado el estudio de las enfermedades de origen priónico. Otras afecciones, tales como las enfermedades de Alzheimer y Parkinson, también se caracterizan por la formación de placas neuronales y podrían estar vinculadas con las enfermedades priónicas.

Lo irónico de estos resultados radica en que la prevención de la progresión de los síntomas de enfermedad de origen priónico podría depender precisamente de una fracción de proteína derivada de priones. Es demasiado pronto para saber la utilidad de esta información en lo referente al tratamiento de la enfermedad, pero cabe esperar que de ella surjan nuevas posibilidades terapéuticas. (National Institute of Allergy and Infectious Diseases. Lab experiments reveal potential therapeutic target for degenerative brain diseases. Comunicado de prensa, 2 de agosto de 1999). 